\title{
A MULTI-AGENT SYSTEM FRAMEWORK FOR SUPPLY CHAIN MANAGEMENT UNDER DISTRICT ALLIANCE SETTING
}

\author{
Fangzhong $\mathrm{Qi}^{1}$, Gengui Zhou ${ }^{1}$, Yongzhu $\mathrm{Qi}^{2}$ \\ ${ }^{l}$ College of Business \& Administration, Zhejiang University of Technology, China; Email: \\ fz_qi@zjut.edu.cn. ${ }^{2}$ Institute of Advanced Manufacturing Technology, Lanzhou University of \\ Technology, Lanzhou 730050, China.
}

Abstract: Multi-agent system (MAS) is regarded as an efficient approach to solving dynamic and distributed problem in complicated operation process of supply chain. The MAS organization architecture for supply chain of district alliance is discussed based on virtual agent federation. A MAS architecture model for supply chain system is created and the functions of facilitator agent and Workflow engine have been emphasized in the model. Multiple upper-layer agent federations are designed to execute the complex system tasks and to implement the system functions. A MAS system framework which has features of high reactivity, reusability, flexibility and reliability is proposed based on CORBA standards. Application example is illustrated in a synergic business prototype system under district alliance setting.

Key words: $\quad$ MAS, Supply Chain Management, District Alliance, System Framework.

\section{INTRODUCTION}

District alliance, a developing manufacturing mode widely practiced in Southeast China, has contributed a lot to Chinese economy during the last decade. In such alliance, production system is a dynamic and complex supply chain system which is organized by several large, almost dominant enterprises and their tightly collaborative partners such as suppliers, manufacturers, distributors. The combination of these independent entities and the number of collaborative partners in a supply chain often change according to market opportunity and business strategic. Highly dynamic environment requires synergic work in supply chain management that provides more accurate allocation of costs to products or activities by

This project is supported by the National High Technology Research and Development Program of China under the grant No. 2002AA414070.

Please use the following format when citing this chapter:

Qi, Fangzhong, Zhou, Gengui, Qi, Yongzhu, 2006, in International Federation for Information Processing (IFIP), Volume 207, Knowledge Enterprise: Intelligent Strategies In Product Design, Manufacturing, and Management, eds. K. Wang, Kovacs G., Wozny M., Fang M., (Boston: Springer), pp. 334-343. 
optimizing inter-organization resources. Therefore, the challenge of today's district alliance is to find novel ways to run their businesses to become more flexible, reliable, and responsive. Agent has emerged as an effective technology to support the management and operation of supply chain $[1,2]$. Considering the features in district alliance, it is reasonable to abstract these independent entities as autonomous agents which deal with business transactions. The integrated supply chain system can be viewed as a multiagent system formed by agents that subject to different organizations.

The structure of any MAS is naturally a distributed one, and the connection between agents is a point-to-point relationship. Such fully distributed structure makes the system to be highly agile, but every agent in the system is required to own more knowledge about its environment. Supply chain faces dynamic and fluctuant market place, and the scope of resource optimization extends to even wider district, this will cause the structure of agent to be more complicated. Along with the number of involved enterprises and entities in supply chain increases, the cost of system communication in such point-to-point network connection will become a serious problem. We adopt the concept of agent federation [3, 4]: the whole supply chain system in a district alliance can be designed as a multi-agent system constructed by cohesive and dynamic agent federations for all business functions of partner enterprises participated in the supply chain.

The organization of agent federation is not unchangeable, but can be dynamically reconstructed according to the changing business process. Combined with the viewpoint of dynamic alliance, we further introduce the concept of virtual agent federation: before a production task or a customer order is put forward, each distributed autonomous agent is on the state of loosely coupling; As soon as system task is confirmed, all agents send their own intention to other agents by their status and interest based on the mechanism of contract-net. For a particular system task, these agents will come into being a virtual agent federation to accomplish the task according to coordination protocols. When system task is completed, the virtual agent federation will disperse automatically and resource will be released waiting for next task. The existence presupposition of virtual agent federation is that each agent owns its superiority and domain knowledge, and can execute determinate business functions.

\section{A MULTI-AGENT SYSTEM ARCHITECTURE MODEL}

The whole supply chain system can be viewed as an autonomous multi- 
agent system which is composed of agents belong to different enterprises, so any node enterprise or organization on a supply chain can be abstracted to a set of agent federations - the lower layer agents in a agent federation can be supervised by the upper layer agents and by contraries, the upper layer agents can specify tasks to the lower layer agents. The agent federation can process nesting according to actual requirements, namely the agent at the middle layer of an agent federation will be the administrator of their lower layer agent and will be supervised by their upper layer agent. Based on above discussion, we created a multi-agent system architecture model for supply chain under district alliance setting, as shown in Figure 1.

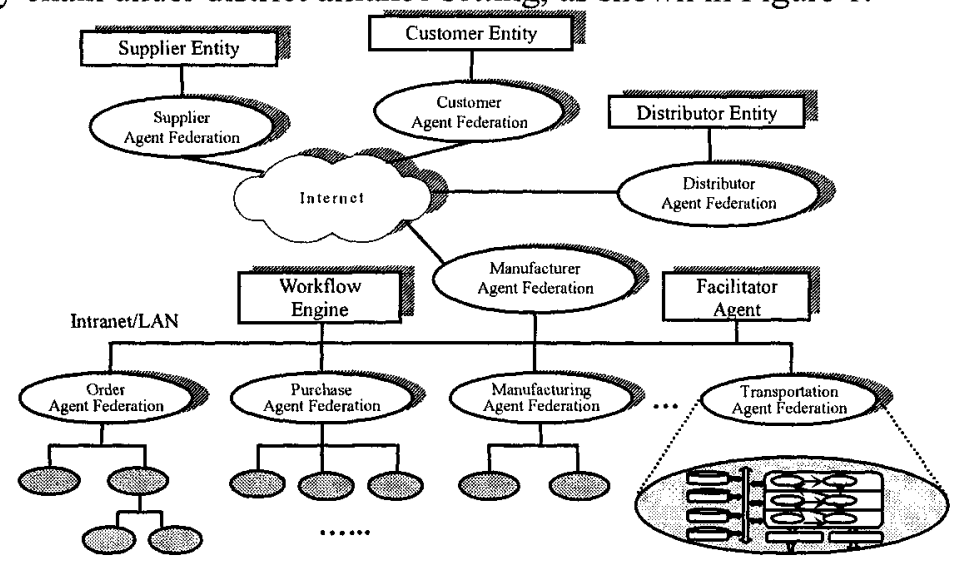

Figure 1. A Multi-Agent System Architecture Model in Supply Chain.

Generally, supply chain is a production mode that aims at a certain market opportunity and is driven by customer orders, so the top layer agent in the federation is usually an order agent. The tasks of an order agent are to administrate the inter-organization cooperative works and to receive, send or monitor customer orders at enterprise level. After an order agent has received an order message from a customer, it queries other agents about the production circumstantiality or capacity to make sure if the order could be promised. As soon as the order has been confirmed, it specifies the task according to the different interest and capability of its lower layer agents. During this process, the work of task distribution could be implemented by adopting one-to-one negotiation or one-to-many auction mechanics. Finally, a feasible task implement plan is set down and the planning result is submitted to work flow engine. Actually, the plan set here is an order execution plan.

While plan is being executed, the work flow engine sends the work task to the work list of every function agent based on order execution plan. Each function agent carries out the practical function operation about the task 
assigned by the work flow engine. After the task has been accomplished, the relevant information is written into process database and the work flow engine does not startup the next task of the function agent accordingly until the order has been fulfilled. Actually, the work flow engine acts as the role of monitoring the process of plan executing within the whole multi-agent system. For exceptional situation such as order postponed or plan readjusted, the plan or activity will be set to the state of suspension. The work flow engine sends request message to the order agent who will negotiate with other functional agent, and this leads to the next negotiation process.

During the process of task distribution, sometimes conflicts between local and overall benefit could occur probably. Under such circumstances, the lower layer agent must submit to the upper layer agent. In regard to the number of layers in an agent federation, it relates to the scale of the system and the granularity of each agent de-signed. Commonly, we think that 2 to 4 layers will be appropriate in practice.

Considering the peculiarities in the operation process of district alliance, the multi-agent system of supply chain we designed is composed of several upper layer agent federations. We define their core functions as followings:

- Order Agent Federation: in charge of accepting customer orders and negotiating with other functional agent about the order production task. It also creates or adjusts order execution plan, deals with exceptional event, customer information maintenance, order auction and negotiation. This federation is the core part of the whole supply chain system.

- Resource Agent Federation: in charge of the dynamic management of all available inner- and inter-organization resource, gathering the capability and status information of all production resource.

- Purchase Agent Federation: in charge of selecting supplier, maintaining the supplier information, creating purchase plan, monitoring the purchase process, and managing account payable. Other functions of this federation include investigating the material and parts or components supplied, inquiring price and inviting public bidding for the material demanding.

- Transportation Agent Federation: in charge of managing the transfer and convey of material within the organization or among collaborative enterprises, functions also include transportation route planning, coalition of transportation task, creating the transportation plan and maintaining the transportation equipment.

- Finance Agent Federation: in charge of inner- or inter-organization cost control and management, including cost accounting, cost planning, cost forecasting and cost analytic Statistics. 
- Manufacturing Agent Federation: in charge of implementing the production task in each workshop or manufacture cell, mainly including the creating of workshop synergic operation plans, scheduling the production task in workshop or work center.

These agent federations are classified by executing functions in the business process of supply chain management. Several children agents could be employed cooperatively to fulfill a certain task under a certain circumstance. For example, the order agent federation could be decomposed to such children agents as customer ser-vice agent, task auction agent, order planning agent. The magnitude of agent granularity to be designed is a problem noteworthy. If an agent granularity is thick enough, the level of system reusability will be getting worse, and will lessen the distributing and autonomous capability of the multi-agent system. Reversely, if an agent granularity is too thin, the complexity of the system will increase accordingly, and the cost of collaborative communication among multiple agents will also increase greatly. During the process of our research, we make detailed plan and system analysis for the supply chain system applied in the district manufacture alliance. Based on this necessary work in advance, the functional agents with appropriate granularity are created to constitute the whole supply chain multi-agent system.

When the collaborative enterprises participated in a supply chain for a certain market opportunity is too many, there is a serious problem about site allocation and capacity matching between service demander and service provider. In the multi-agent system of supply chain, we introduce a third part agent called facilitator or match-maker $[5,6]$ whose main task is to play the role of bridge over the service provider and service demander by abstracting and describing the capability of information service it owned. The service provider registers its capacity to the facilitator agent. As any service demander makes a query, the facilitator will match these two parts, and the facilitator's task is finished after sending the matching information to the service demander. The multi-agent system is configured with several facilitator agents to solve the site allocation and capacity matching problems among multiple agents. The introducing of facilitator provides an agile and effective interactive channel in the communication between agents.

\section{MULTI-AGENT SYSTEM SOFTWARE FRAMEWORK}

From the view of system design, the action of agent is to implement a system function call. As there are many Legacy systems running in different collaborative enterprises inevitably, we encapsulate the modules to be called by each agent by employing CORBA standard. The whole software system 
is composed of Legacy system, CORBA middleware, CORBA objects and agent/agent federation.

Figure 2 illustrates a multi-agent software framework in the supply chain of district alliance. The structure of the whole system is a distributed architecture based on CORBA mechanism. Because the application service based on CORBA represents to the outside as an object-oriented interface definition which employs the interface definition language (IDL) to define, the co-operation between application services can be independent from location and environment. These application services will be convenient to implement the action of agents. Application to be developed should act in accord with CORBA standards, and the final application designed will be either a CORBA object or an application server which can provide remote call service. As for Legacy system, we introduce a system encapsulation method called Adapter to encapsulate the Legacy system forming the CORBA object or application server with the remote call function.

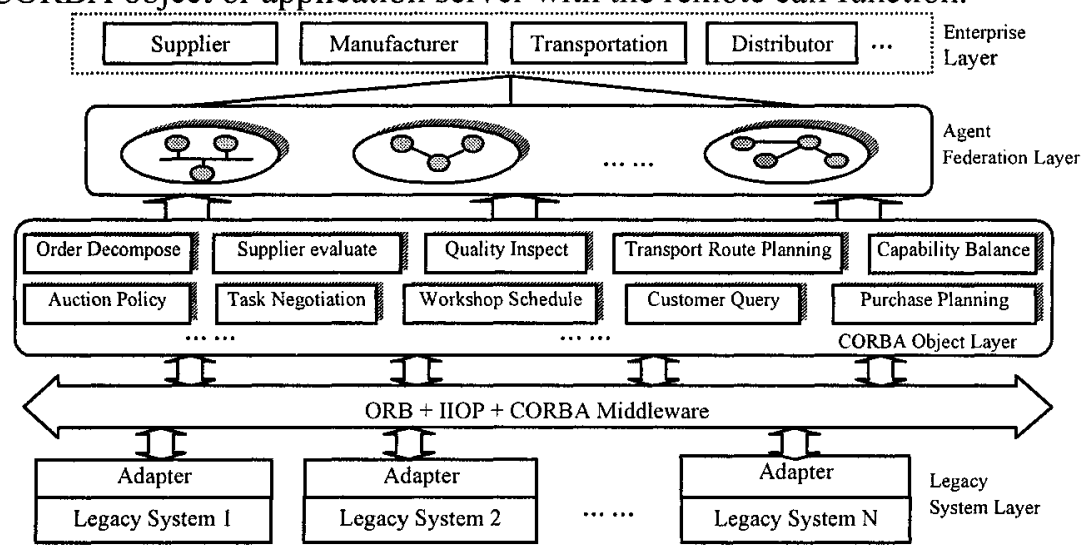

Figure 2. System Software Framework of Multi-Agent System in Supply Chain.

The supply chain multi-view models created at our initial system analysis phase has been used to guide the designing of the CORBA application layer and agent/agent federation application layer of the multi-agent system by using information encapsulation techniques. By adopting such hierarchy framework and integrated multi-view models, it will accelerate the establishment of application and operation environment in the supply chain system design [7]. The modeling idea of the supply chain system runs through the creating of system application objects and the designing of agent - any changing about the multi-view model merely impacts on the changing of the action or function of the agent. Above the CORBA object is the agent federation layer - they can call or communicate with each other. Several 
relevant agents compose agent federation, and the agent federations represent the enterprise functions which participate in the implementation and operation of the supply chain system.

The upper layer agent federations which are designed based on dynamic alliance concepts, can be created, adjusted and reconstructed in response to the market demand and to the optimization of operation process and organization. It can plan, schedule and manage the main process of collaborative work in the operation of supply chain management. By negotiating and adjusting adequately among these upper layer agents, organizations in the supply chain system could contact suppliers or customers as earlier as possible. To optimize every process in the supply chain system, they can also provide the chance to discover problems and take remediation action as soon as possible. The steps in process and subroutines that involves fewer steps are dominated by lower layer agent federations, so the functions of lower agents are relatively steady. This will increase the response speed to market, increase the system reliability and capability of reuse. When the market opportunity is fulfilled and the supply chain is decomposed, the reengineering of the supply chain can be realized quickly by re-configuring the agents' or agent federations' functions according to new business process. The implementation of agent employs CORBA middleware techniques, so each heterology agent is provided with the feature of plug and play. Ideally, if all processes in the supply chain system employ the same techniques, information in the supply chain system will realize real seamless integration, what needs to do is just adjusting the agents' functions to reconstruct the supply chain based on the actual requirement.

\section{CASE STUDY-AN EXAMPLE}

Based on the above discussion, we have designed a district allianceoriented supply chain system model and developed a prototype system. The system includes sales agents ES_Agent1 and ES_Agent2 which represent the suppliers in the system, purchase agent $P_{-}$Agent, manufacture agent M_Agent, sales agent S_Agent, transportation agent T_Agent and the enterprise Workflow management system (WfMS). The architecture of the distributed multi-agent system is shown in Figure 3.

Each agent in the system is programmed in Java, and the connection between Java and database employs JDBC_ODBC Bridge. Among these

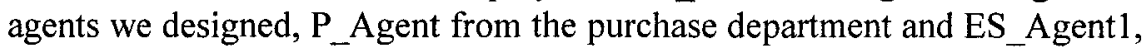
ES_Agent2 from suppliers apply the technology of mobile agent. These mobile agents have been developed on the Aglets development platform 
which is provided by IBM Corporation. The main process of the multi-agent system is divided into two phases:

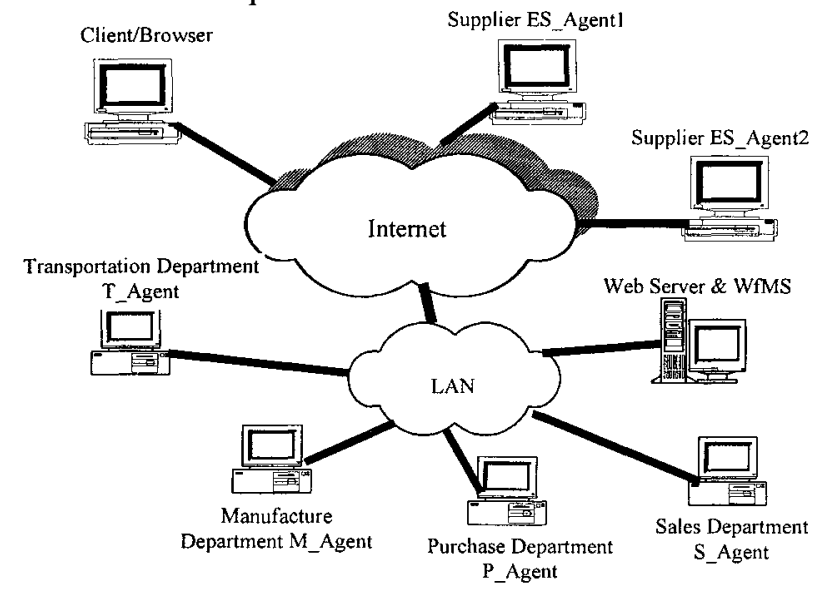

Figure 3. Distributed System Architecture of MAS in Supply Chain.

1. The confirmation of customer order and the process of negotiation;

2. The actual production process.

Customers will obtain interrelated data through their interaction with the web server firstly. If a new order is created, the request message of new order is sent and the sales department's S_Agent will be activated. Then S_Agent will query the inventory about finished product based on the material code of the product, and if the quantity on hand is enough, it will send transportation message to the T_Agent. Its task of this new order will be finished as soon as its data item is updated. If the quantity is not enough, S_Agent will calculate the net amount of requirement, and then it will send the product style, quantity and date of delivery to the manufacture department's M_Agent. M_Agent will then confirm whether it can finish the task based on its capability. If there is any conflict, a feasible solution will be found by sufficient negotiation between M_Agent and S_Agent at this stage. Subsequently, the result of negotiation will be sent to purchase department's P_Agent. After P_Agent gets the production message from M_Agent, it will decompose the task based on BOM and combine the raw and processed materials to produce a purchase bill. Then the P_Agent will verify the requirements to see if they could be satisfied and if there is any conflict, it will solve the problem by negotiating with M_Agent. By contraries, it will send confirmation message to M_Agent. As soon as M_Agent gets the confirmation message, it will send confirmation message to $S_{-}$Agent. Thus, 
the whole order confirmation process is finished, and $S_{\text {_Agent }}$ will mark the order confirmation as ready to the Workflow management system (WfMS).

The production process is driven by the Workflow management system [8]. Each work process and the assigning of executive role to each process have been predefined in WfMS, and here we realize this by the method of database table. The production process of the order is started up by system administrator at an appointed time. The basic process is: purchase, quality inspect, in and out of inventory, parts manufacture, components assemble and finished product in stock.

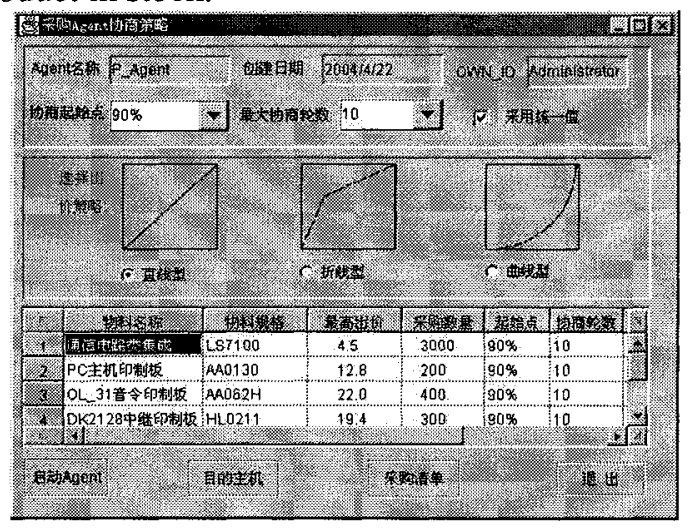

Figure 4. Negotiation Policy of the Mobile Agent P_Agent.

During the process of purchasing, we introduce the mode of mobile agent. The mobile agent can move from local host to the suppliers' host according to the suppliers' IP addresses which the purchase staff has provided. The mobile agent communicates with the suppliers' ES_Agent1 and ES_Agent2 to get the quoted price of raw materials. After comparing the quoted price of same material from the two suppliers, the quotation will be fetched to the local host. Then the purchase staff is able to fix on a supplier and execute the further actions in the purchasing process.

After the purchase process of raw and processed materials has finished, P_Agent marks the purchase accomplishment as ready to the WfMS. The WfMS then sends messages to M_Agent and T_Agent sequentially according to the predefined process. Figure 4 shows the confirmation process about the negotiation policy of the mobile agent $P_{-}$Agent.

\section{CONCLUSION}

The MAS framework we designed provides a supply chain system with the feature of high reconstruction. While outside environment of the system is changing, the only work we need to do is to adjust the functions of the 
relevant agent or to let the agent join in or leave from the system automatically according to the actual requirement, and this will not affect the natural work of any other agent within the system. Furthermore, these agents could exchange information directly with the Legacy system at the interface layer through the interface definition of Legacy system based on CORBA standards. Such practice shortens the cycle of system development because we need not to redesign the whole system. As the agents are designed in conformity to the intelligent models of different functions within the different enterprises' problem domains, they are able to respond continuously to the ever-changing environment while they are executing a certain tasks the system designers have defined under the dynamic circumstances, if the conditions are satisfied. By employing the agent technology, the integrated scope of enterprise application system could be extended to either upper or downstream cooperative partners. At the same time, these agents have the characteristic of high reactivity, so multi-agent system can answer incidents such as cancelled order or client system breakdown - these will reduce the enterprise's losing farthest.

\section{REFERENCES}

1. Jin, D., Yueting, C. and Jiaben, Y. Coordination and Optimization in Agile Supply Chain Management Based on Multiple Agent System. System Engineering - Theory \& Practice, Vol. 20, No. 8, Aug. (2000), pp. 7-11

2. Teigen, R. Information Flow in a Supply Chain Management System. Diploma Thesis. Univer-sity of Toronto, May (1997)

3. Hai, Z., Jian, C., Yulin, F. and Xiaoqing, S. A Federation-Agent-Workflow Simulation Framework for Virtual Organisation Development. Information \& Management, Vol. 39, No. 4, Jan. (2002), pp. 325-336

4. Khedro, T. A Distributed Problem-Solving Approach to Collaborative Facility Engineering. Advances in Engineering Software, Vol. 25, No. 2-3, March-April (1996), pp. 243-252

5. Fischer, K. The Design of an Intelligent Manufacturing System. In Proceedings of the 2nd International Working Conference on Cooperating Knowledge-based Systems, University of Keele, 1994, pp. 83-99

6. Finin, T., Fritzon, R. McKay, D. and McEntire, R. KQML - A Language and Protocol for Knowledge and Information Exchange. Tech. Report, University of Maryland, Baltimore, 1993

7. Zhigang, Z., Xiaohong, P., Ming, G. and Yaodong, C. Research on Modeling Technology of Agile Supply System Supporting Dynamic Alliance. China Mechanical Engineering, Vol. 12, Suppl. 1, May (2001), pp. 64-67

8. Fangzhong, Q., Zhengxiao, W., Xiaohong, P., Yaodong, C. and Zhigang, Z. Research on Workflow Management System (WfMS) Framework in Agile Supply Chain. China Mechanical Engineering, Vol. 13, No. 9, May (2002), pp. 750-755 